\title{
Seismic anisotropy changes across upper mantle phase transitions
}

\author{
Kaiqing Yuan ${ }^{\mathrm{a}}$, Caroline Beghein ${ }^{*, \mathrm{a}, 1}$ \\ ${ }^{a}$ University of California Los Angeles, Department of Earth and Space Sciences, 595 \\ Charles Young Drive East, Box 951567, Los Angeles, CA 90095-1567, United States
}

\section{Abstract}

The mantle transition zone is believed to play an important role in the thermochemical evolution of our planet and in its deep water cycle. Constraining mantle flow at these depths can help elucidate its nature and better understand mantle dynamics and the history of plate tectonics. Seismic anisotropy, i.e. the directional dependence of seismic wave velocity, provides us with the most direct constraints on mantle deformation. Its detection below $\sim 250 \mathrm{~km}$ depth is challenging, and it is often assumed that the deep upper mantle is seismically isotropic due to a change in mantle deformation mechanism. Here, we present a global model of azimuthal anisotropy for the top $1000 \mathrm{~km}$ of the mantle. We used a dataset composed of fundamental and higher mode Rayleigh wave phase velocity maps, which provides resolution of azimuthal anisotropy to much greater depths than in previous studies. Our model unravels the presence of significant anisotropy in the transition zone, challenging common views of mantle deformation mechanisms, and reveals a striking correlation between changes in anisotropy amplitudes and in the fast

\footnotetext{
*Corresponding author

Email address: cbeghein@ucla.edu (Caroline Beghein)
} 
direction of wave propagation where upper mantle phase transitions occur. The newly found relation between anisotropy changes and phase transformations gives new insight on the nature of the MTZ and suggests that the anisotropy originates from the lattice preferred orientation of anisotropic material. While the interpretation of our results in terms of mantle deformation is not straightforward due to many possible scenarios, possible explanations for our findings include recrystallization during phase transformations, or changes in the slip system across the MTZ boundaries, which in turn could be explained by changes in the water content of mantle material, consistent with the idea that the transition zone acts as a water filter.

Key words: Transition zone, Anisotropy, Surface waves, Seismology, Geodynamics, Mineral Physics

\section{Introduction}

The mantle transition zone (MTZ) is defined by discontinuities in seismic wave velocities at $410 \mathrm{~km}$ and $670 \mathrm{~km}$ depth. It has long been at the center of the debate between multi- and single-layered circulation models that relates to heat transport and chemical mixing throughout the mantle (Anderson, 1967; Schilling, 1973; Hager and O'Connell, 1979). It was also suggested that the MTZ is a reservoir that collects water transported by subduction of the lithosphere into the mantle (Bercovici and Karato, 2003). Constraining mantle flow at these depths is thus essential to get insight on the nature of the MTZ and to better understand mantle dynamics and the history of plate tectonics.

The seismic discontinuities that delimit the MTZ are traditionally in- 
terpreted as being dominated by pressure-induced phase transitions of the olivine structure into wadsleyite at $410 \mathrm{~km}$ depth and from ringwoodite to perovskite and magnesiowustite at $670 \mathrm{~km}$ depth (Anderson, 1967). Seismic reflection and refraction studies can constrain the depth, size, and sharpness of the associated velocity jumps, which can be compared to experimental data to infer constraints on the thermal and chemical nature of the mantle (Shearer, 2000). These analyses are typically performed using isotropic velocities and therefore do not account for seismic anisotropy. Seismic anisotropy is however a powerful tool that gives us a more complete description of mantle material elasticity, and that can provide some of the most direct constraints on mantle flow patterns since it can result from deformation by dislocation creep during convection (Karato and $\mathrm{Wu}, 1993$ ).

Two types of anisotropy can manifest themselves in seismic data: radial or polarization anisotropy, describing whether the vertical or horizontal direction is seismically faster, and azimuthal anisotropy, which is the dependence of seismic wave-speed within a horizontal layer. Observations of global and regional seismic anisotropy are abundant in the top $\sim 200-250 \mathrm{~km}$ of the mantle (e.g., Montagner and Kennett (1996); Debayle et al. (2005); Panning and Romanowicz (2006); Yuan and Romanowicz (2010)). Lattice preferred orientation (LPO) of olivine is often invoked to explain shallow mantle seismic anisotropy because it is the most abundant mineral, it has high single-crystal anisotropy (Mainprice et al., 2005), and large individual mineral grains can develop a fabric under high stress, i.e., in the dislocation creep regime (Karato, 1992). Olivine LPO in the lithosphere is often interpreted in term of past tectonic events (Nishimura and Forsyth, 1989), and 
asthenospheric anisotropy is regarded as a manifestation of current mantle deformation because the fast direction of wave propagation tends to align with the present-day absolute plate motion (APM) at these depths (Marone and Romanowicz, 2007b).

The detection of seismic anisotropy in the deep upper mantle remains however elusive. Large discrepancies among studies (Montagner and Kennett, 1996; Beghein and Trampert, 2004b; Beghein et al., 2006; Panning and Romanowicz, 2006; Visser et al., 2008b) cast doubt on the ability of intrinsically anisotropic mantle material to align in convective flow at large depths (Karato, 1992). It was also suggested that only the Australian plate displays significant azimuthal anisotropy below $175 \mathrm{~km}$ depth, and that anisotropy under other continents is shallower and only weakly correlated to presentday APM (Debayle et al., 2005). These particular results have since been challenged by regional investigations of the north American upper mantle. These more recent studies, which benefited from better data coverage than the earlier work, unraveled anisotropy in the asthenosphere (Marone and Romanowicz, 2007b) and possibly deeper (Kosarian et al., 2011). Multiple global models indicate the presence of radial anisotropy in the MTZ (Montagner and Kennett, 1996; Beghein and Trampert, 2004b; Beghein et al., 2006; Panning and Romanowicz, 2006; Visser et al., 2008b), but uncertainties are large (Beghein et al., 2006). Seismic anisotropy has also been detected inside and below the MTZ using shear-wave splitting (Fouch and Fischer, 1996), but few global models display significant azimuthal anisotropy at these depths. Global MTZ azimuthal anisotropy has been shown to be compatible with long-period Love waves (Trampert and van Heijst, 2002) and coupled free 
oscillations (Beghein et al., 2008). However, these studies were no more than tests to estimate the likelihood of MTZ anisotropy, and well-resolved global three-dimensional (3-D) models have been lacking thus far.

The main objective of this study was to model 3-D azimuthal anisotropy in the top $\sim 1000 \mathrm{~km}$ of the mantle to determine whether seismic anisotropy is present at MTZ depths and to add new constraints on deep upper mantle deformation. For this purpose we employed a dataset composed of the previously published azimuthally anisotropic phase velocity maps of Visser et al. (2008a) constructed for fundamental modes and overtone Rayleigh waves. Modeling seismic anisotropy below $\sim 250 \mathrm{~km}$ depth has been challenging because the vertical resolution of most datasets decreases with depth. For instance, shear-wave splitting data are integrated measurements along quasivertical paths and have therefore poor depth resolution. In addition, many surface wave studies use fundamental modes only, which can only resolve the upper $\sim 250 \mathrm{~km}$ of the mantle for commonly used periods of $50 \mathrm{~s}$ to $200 \mathrm{~s}$. The data employed here are sensitive to much greater depths than in most previous studies and are thus well suited to get new insight on deep upper mantle circulation.

\section{Data}

The data employed in this study were the degree 20 anisotropic phase velocity maps of Visser et al. (2008a) for Rayleigh wave fundamental modes and the first six overtones at periods comprised between 35 and $175 \mathrm{~s}$ (see Fig.1 caption for details). Owing to their dispersive properties surface waves are ideal to put depth constraints on seismic anisotropy and velocities. How- 
ever most previous global surface wave inversions of azimuthal anisotropy have been limited to fundamental modes only, which cannot resolve structure beyond $\sim 250 \mathrm{~km}$ depth (Nishimura and Forsyth (1989); Montagner and Tanimoto (1991); Debayle et al. (2005)). By using the fundamental modes and overtone data we extended the resolution to the deep upper mantle and top of the lower mantle (Fig. 1).

Visser et al. (2008a) performed their automated dispersion measurements using a model space search approach, which led to more measurements of higher modes than previously published. A difficulty in measuring higher modes is the separation of the overtones. The forward modeling method they employed enabled them however to determine the statistical significance of their measurements for the different modes, i.e., they were able to determine the number of higher modes reliably constrained by the seismograms. Another advantage of their method was that it provided consistent phase velocity uncertainties for all the modes measured.

The authors inverted their path-averaged measurements to construct global anisotropic phase velocity maps. In a slightly anisotropic medium, surface wave phase velocity perturbations $d c$ at a given point on Earth's surface can indeed be expressed as (Smith and Dahlen, 1973):

$$
\begin{aligned}
d c(T, \Psi)=d c_{0}(T) & +d c_{1}(T) \cos (2 \Psi)+d c_{2}(T) \sin (2 \Psi) \\
& +d c_{3}(T) \cos (4 \Psi)+d c_{4}(T) \sin (4 \Psi)
\end{aligned}
$$

where $\mathrm{T}$ is the period of the wave, $\Psi$ is the azimuth of propagation, and $d c$ is expressed with respect to a one-dimensional (1-D) reference model. $d c_{0}$ is the phase velocity perturbation averaged over all azimuths and $d c_{i}(i=$ $1, \ldots, 4)$ are anisotropic terms that describe the azimuthal dependence of the 
phase velocity. In our study we used the $2 \Psi$ anisotropy terms of these phase velocity maps to model 3-D azimuthal variations in vertically polarized shearwave velocity $\left(V_{S V}\right)$. Visser et al. (2008a) verified that the phase velocity azimuthal dependence was required by the data and robust within estimated data uncertainties for all the modes considered. The $2 \Psi$ anisotropy was even shown to be robust within two standard deviations.

Resolution of the phase velocity maps can be affected by the quality of the data path azimuthal coverage. Visser et al. (2008a) showed that the coverage was very good everywhere for the fundamental modes, yielding little trade-offs between the isotropic and anisotropic terms of equation 1. The number of measured modes decreased with the overtone number, therefore decreasing the ray coverage, especially in the southern hemisphere. This is especially visible in their Figure 1 for the fifth and sixth higher modes in the southeast Pacific, southern Indian ocean, and part of the Atlantic. In continental regions and northwestern and central Pacific the ray coverage is still very good for the higher modes, giving us confidence in the azimuthally anisotropic part of the phase velocities in these regions.

The choice of the damping affects resolution as well. The authors chose an overall damping such that the relative model uncertainty remains constant for all modes. This resulted in phase velocity maps of decreasing resolution with increasing overtone number. Visser et al. (2008a) estimated that on average the $2 \Psi$ terms of the fundamental mode phase velocity maps have a resolution comparable to that of a spherical harmonic expansion of degree and order 8 , and the $2 \Psi$ terms of the higher modes have decreasing resolution down to degree 5 . This implies a resolving power of about $5000 \mathrm{~km}$ near the 
surface, decreasing to $\sim 8000 \mathrm{~km}$ at MTZ depths. Using data with varying resolution is not ideal for depth inversions. However, the inferences made in this paper focus on large-scale anisotropy only. The change in resolution with the type of mode should therefore not strongly affect our results.

Another common source of uncertainty when constructing anisotropic phase velocity maps is the existence of trade-offs between the isotropic (i.e., azimuthally independent) and anisotropic terms of equation 1, which can result in lateral heterogeneities being mapped into the anisotropy. However, with the calculation of resolution matrices Visser et al. (2008a) showed that there was little mapping of isotropic structure into the anisotropy. We can thus safely invert the terms of equation 1 independently of each other and infer information on Earth's 3-D anisotropy from the models obtained.

\section{Methods}

\subsection{Forward and inverse problem}

We used the $2 \Psi$ anisotropic terms of equation 1 to constrain 3-D $V_{S V}$ azimuthal variations in the top $1000 \mathrm{~km}$ of the mantle. The inversion of the isotropic term $d c_{0}$ was performed by Visser et al. (2008b) using Love and Rayleigh wave data, and yielded a 3-D model of velocity and radial anisotropy showing that global radial anisotropy with $V_{S V}>V_{S H}$ is likely present inside and below the MTZ with an amplitude between 1 and $2 \%$. Our results are complementary to those previous inferences as they provide constraints on different elastic parameters (Montagner and Nataf, 1986).

We used a singular value decomposition method to solve the inverse problem and followed Matsu'ura and Hirata (1982) to determine how many singu- 
lar values to keep in the reconstruction of the model. We inverted the following equations that relate phase velocity anisotropy to azimuthal anisotropy at depth:

$$
\begin{aligned}
d c_{1}(T) & =\int\left[G_{c}(r) K_{G}(T, r)+B_{c}(r) K_{B}(T, r)+H_{c}(r) K_{H}(T, r)\right] d r \\
d c_{2}(T) & =\int\left[G_{s}(r) K_{G}(T, r)+B_{s}(r) K_{B}(T, r)+H_{s}(r) K_{H}(T, r)\right] d r
\end{aligned}
$$

where elastic parameters $G_{c}(r)$ and $G_{s}(r)$ relate to $V_{S V}$ azimuthal anisotropy, and the other four parameters relate to P-wave azimuthal anisotropy (Montagner and Nataf, 1986). $K_{G}(r, T), K_{B}(r, T)$, and $K_{H}(r, T)$ are the local partial derivatives, or sensitivity kernels, for Rayleigh wave at period $\mathrm{T}$ and radius $\mathrm{r}$, which can be calculated for a given reference model using normal mode theory (Takeuchi and Saito, 1972). The kernels were calculated using model PREM (Dziewonski and Anderson, 1981) for all the modes used here (Fig. 1 and Supplementary Fig. 1).

The fast azimuth of propagation $\Theta$ and the anisotropy amplitude $G$ of vertically polarized shear-waves are given by:

$$
\Theta=\frac{1}{2} \arctan \left(G_{s} / G_{c}\right)
$$

and

$$
G=\sqrt{G_{s}^{2}+G_{c}^{2}}
$$

Similarly, $B=\sqrt{B_{s}^{2}+B_{c}^{2}}$ and $H=\sqrt{H_{s}^{2}+H_{c}^{2}}$. $B$ and $H$ are however affected by large trade-offs due to the similarity of their partial derivatives (Supplementary Fig. 1), and are therefore poorly resolved. While some researchers choose to neglect them and invert surface wave data for $G$ only, we solved equations 3 and 4 exactly for all three parameters. We presented 
the $B$ and $H$ models in section 4.1 for completeness, but we mostly focus this manuscript on the best-resolved parameter $G$. Equations 3 and 4 were inverted independently and the $G_{c}$ and $G_{s}$ models were combined to obtain $G$ and $\Theta$. We verified a posteriori that little trade-offs exist between $G$ and these other parameters (Supplementary Fig. 2), and we performed tests which showed that that the data require $B$ and $H$ to be included in the inversion, and that our results are reliable (Supplementary material).

\subsection{Parametrization}

We divided Earth's surface into $2^{\circ} \times 2^{\circ}$ cells, and inversions of equations 3 and 4 were carried out at each grid cell. This cell parametrization was designed to follow the same grid as that of crustal model CRUST2.0 (Bassin et al., 2000), which we employed to account for crustal structure (section 3.3). Of course, the lateral resolution of our model is directly controlled by the resolution of the phase velocity maps and is limited to larger wavelengths than the precision of our grid. To insure that we do not interpret features of the model that are not resolved by the data, the $2^{\circ} \times 2^{\circ}$ model obtained was expanded in generalized spherical harmonics (Phinney and Burridge, 1973) and filtered up to degree and order 20 to match the spherical harmonic expansion of the phase velocity maps used here.

At every grid cell we parameterized $G_{c}(r)$ and $G_{s}(r)$ vertically using 18 cubic spline functions $S_{i}(r)(\mathrm{i}=1, \ldots, 18)$ of varying depth spacing (Supplementary Fig. 3), similarly to Visser et al. (2008b) who inverted the isotropic component of the dataset used here:

$$
G_{c}(r)=\sum_{i=1}^{18} G_{c}^{i} S_{i}(r)
$$




$$
G_{s}(r)=\sum_{i=1}^{18} G_{s}^{i} S_{i}(r)
$$

An identical depth parametrization was used for $B_{c}(r), B_{s}(r), H_{c}(r)$, and $H_{s}(r)$.

\subsection{Crustal corrections}

The previous generation of 3-D velocity and anisotropy models obtained from surface wave inversions used sensitivity kernels calculated based on the 1-D reference mantle model PREM (Dziewonski and Anderson, 1981). It was however demonstrated that not accounting for 3-D variations in crustal structure and their effect on the partial derivatives can affect 3-D mantle models (Boschi and Ekström, 2002; Marone and Romanowicz, 2007a; Kustowski et al., 2007; Bozdağ and Trampert, 2010). Recent studies have performed more accurate crustal corrections, thereby reducing the mapping of crustal structure into the deep mantle (Beghein and Trampert, 2004a; Panning and Romanowicz, 2006; Kustowski et al., 2008; Nettles and Dziewonski, 2008; Visser et al., 2008b; Beghein, 2010; Ferreira et al., 2010; Yuan and Romanowicz, 2010). Here we adopted an approach similar to that of Boschi and Ekström (2002) and determined a set of laterally varying partial derivatives calculated on our $2^{\circ} \times 2^{\circ}$ grid. At each grid cell we generated a local 1-D model composed of crustal model CRUST2.0 (Bassin et al., 2000) on top of the PREM mantle, and calculated the corresponding partial derivatives.

Examples of kernels and how they are affected by crustal structure are displayed in Supplementary Fig. 4. In that example we selected a local crustal model corresponding to Tibet because this is where the Moho depth differs the most from the PREM Moho depth, and therefore where we expect the 
strongest effect on the partial derivatives. The choice of the crustal model affects the sensitivity kernels of fundamental mode Rayleigh waves as expected considering that they are sensitive to relatively shallow structure, but it also affects the sensitivity of the overtones. The effect on the overtone sensitivity amplitude is the strongest near the surface, and we find a $10-20 \mathrm{~km}$ vertical shift in the peaks of the sensitivity curves at larger depths as well. This confirms results from a previous study on shear-wave velocity and radial anisotropy partial derivatives (Marone and Romanowicz, 2007a).

\section{Results}

\subsection{The model}

Our results unveil the 3-D pattern of azimuthal anisotropy in the upper $1000 \mathrm{~km}$ of the mantle and reveal a striking correlation between changes in anisotropy and the location of upper mantle phase transitions. Our root mean square (rms) model amplitude (Fig. 2(a)) has a strong peak of $2 \%$ anisotropy at $100 \mathrm{~km}$ depth, consistent with recent global models (Debayle et al., 2005). Noteworthy are the three broader peaks at $\sim 300 \mathrm{~km}$, inside the MTZ, and around $800 \mathrm{~km}$ depth with $\sim 1 \%$ anisotropy, comparable to amplitudes of radial anisotropy in the deep upper mantle (Beghein et al., 2006; Panning and Romanowicz, 2006; Visser et al., 2008b). Most interestingly, distinct amplitude minima occur in the uppermost mantle and at the MTZ boundaries (Fig. 2(a)), and they are remarkably correlated with high vertical gradients $d \Theta / d r$ in fast propagation direction (Fig.2(b)). Another, smaller peak in $d \Theta / d r$ is visible in the middle of the MTZ, approximately where one would expect the wadsleyite to ringwoodite phase transition to occur. The 
average $B$ and $H$ models are shown in Supplementary Fig. 5 for completeness, but they should not be taken at face value since they are affected by large trade-offs.

Fig. 3 illustrates 3-D azimuthal variations in $V_{S V}$ and confirms that the fast axes change across the MTZ boundaries at most locations. The anisotropy pattern of the shallow mantle is consistent with several features of other global studies (Nishimura and Forsyth, 1989; Montagner and Tanimoto, 1991; Debayle et al., 2005) : fast axes are perpendicular to most spreading ridges, they follow the convergence direction of the Nazca plate under South America, they are roughly oriented southwest-northeast under the Australian plate at $200 \mathrm{~km}$ depth, and north-south in northern Africa. There is also a good alignment between $\Theta$ and the present-day APM in the uppermost mantle (Supplementary Fig. 6) as seen in other models (Debayle et al., 2005). The APM was calculated using HS3-NUVEL 1A in the no-net rotation reference frame (Gripp and Gordon, 2002).

Additionally, Fig. 4 shows that the best alignment between $\Theta$ and the APM is found beneath cratons around $250 \mathrm{~km}$ depth, and beneath oceans over depth ranges that vary with crustal ages. The fast azimuth $\Theta$ is subparallel to the APM over the top 200-250 km for young oceans, and progressively changes as age increases. For oceans older than $80 \mathrm{Ma}$, the alignment with the APM is best between about $150 \mathrm{~km}$ and $250 \mathrm{~km}$ depth. Supplementary Fig.7 compares different APM models and demonstrates that HS3-NUVEL $1 \mathrm{~A}$ in the no-net rotation reference frame generally yields a better agreement with the fast axes than others. 


\subsection{Effect of reference mantle model and synthetic tests}

We first verified that the presence of a discontinuity at $220 \mathrm{~km}$ in the reference model does not affect the results by smoothing the sensitivity kernels and performing new inversions. We also tested whether the changes in anisotropy amplitude and the higher $d \Theta / d r$ found at the MTZ boundaries could be artifacts resulting from the MTZ discontinuities included in the reference mantle model. We first checked that the amplitude minima in $G / L$ are not due to the presence of MTZ discontinuities in the reference $L$ model by plotting the rms amplitude of absolute parameter $G$ as a function of depth (Supplementary Fig. 8).

Using synthetic tests we further investigated whether the discontinuities present in the sensitivity kernels at $410 \mathrm{~km}$ and $670 \mathrm{~km}$ depth could be causing the observed features (Fig. 5 and Supplementary Figs. 9 and 10). These synthetic tests were also used to verify how well our inversion recovers a given input model, with the caveat that they do not account for lateral variations in ray coverage since the data error bars employed in this study are uncertainties averaged over the sphere, as explained below (section 4.5). The reader should also keep in mind that these tests were conducted using sensitivity kernels calculated based on PREM and assuming $B=H=0$. The tests of Fig. 5 revealed that the input model is generally well recovered with the chosen regularization, and Fig. 5(f) demonstrated that the observed low amplitude of anisotropy found in our model at $410 \mathrm{~km}$ and $670 \mathrm{~km}$ depths is not an artifact due to the presence of discontinuities in the reference model and in the sensitivity kernels. Supplementary Fig. 9 displays a synthetic test for the relative anisotropy amplitude $G / L$, and shows that the amplitude is 
very well recovered in the top $700 \mathrm{~km}$. Around $800 \mathrm{~km}$ depth, the recovered amplitude is lower than in the input, and we lose most of our depth resolution beyond that depth. This test also shows that if the input model does not have amplitude minima at $410 \mathrm{~km}$ and $670 \mathrm{~km}$ depth, they do not appear in the output model. Supplementary Figs. 10 and 11 are synthetic tests designed to assess the ability of our data to resolve several layers of anisotropy. In Supplementary Fig. 10 the layering is simulated by an input amplitude displaying a peak and a change in the fast axis $\Theta$ every $200 \mathrm{~km}$. In this case, the angle and amplitude are very well recovered in the top $700-800 \mathrm{~km}$, but not deeper. In Supplementary Fig. 11 the input model displays a peak every $100 \mathrm{~km}$ and a $90^{\circ}$ change in the fast axis in every layer. Both amplitude and fast axis are well-resolved in each layer in the top $400 \mathrm{~km}$. The recovered amplitude is lower than its input value between 400 and $700 \mathrm{~km}$ depth and some vertical smearing of the peak amplitude is visible below that depth. We also observe a slight downward shift of the recovered fast axis compared to the input model below $600 \mathrm{~km}$ depth.

\subsection{Effect of 3-D crustal structure}

In this study we accounted for the effects of 3-D crustal structure on the sensitivity kernels. It should nevertheless be noted that the resulting 3-D model also depends on the accuracy of the crustal model used. In order to test the dependence of our results on the choice of the crustal model, we performed a second set of inversions using partial derivatives based on PREM alone, and compared the new model with the model obtained using laterally varying kernels. Uncertainties on model CRUST2.0 are unknown, but they are likely smaller than the difference between the PREM crust and 
CRUST2.0. The difference between the two models should thus constitute an upper limit on model errors owing to uncertainties in crustal structure.

While small differences were found in the 3-D anisotropy, the two sets of kernels led to equivalent results regarding the average $\mathrm{G} / \mathrm{L}$ and $d \Theta / d r$. Supplementary Fig. 12 shows that using the PREM crustal model led to some changes in the anisotropy rms amplitude, but little differences were found in $d \Theta / d r$, and the two models correlated well above the $95 \%$ significance level. Our inferences on the average anisotropy amplitude and fast direction are thus stable with respect to crustal structure. This is similar to a previous study of velocity and radial anisotropy amplitudes (Marone and Romanowicz, 2007a). The authors showed that crustal structure can affect the 3-D velocity model amplitude in the deep upper mantle, but that the 1-D average model structure is stable and does not strongly depend on the crustal correction.

Similarly, we verified that the effect of 3-D mantle structure does not affect the resulting model (Supplementary Material).

\subsection{F-tests}

Findings of a previously unseen signal of seismic anisotropy in the MTZ need to be accompanied by additional tests to assess whether the data employed truly require deep upper mantle anisotropy. By allowing the model to include anisotropy in the MTZ and below, the data fit improved, but we also increased the number of parameters compared to a model that is isotropic below $410 \mathrm{~km}$ or $670 \mathrm{~km}$ depth for instance. Models with more parameters are more efficient at explaining the data, but the decrease in misfit is not always significant and warranted by the data. Statistical F-tests (Bevington and Robinson, 2002) give the level of confidence with which the differences 
between two misfits are significant. They enable us to compare two inversions obtained with different regularizations and to determine whether the data fit improvement of our model (thereafter referred to as YB13) is mostly due to the increase in the number of parameters and random fluctuations in the data or whether deep anisotropy is constrained by the data.

We performed additional inversions of our dataset in which we imposed the anisotropy to remain above the $410 \mathrm{~km}$ depth (model 1) and above $670 \mathrm{~km}$ depth (model 2). We conducted F-tests to compare the misfit of each of these new models with YB13. The root mean square amplitudes of the three models are represented in Supplementary Fig. 14(a). We employed the reduced $\chi^{2}$ to measure the data misfit (Trampert and Woodhouse, 2003) :

$$
\chi^{2}=\frac{1}{N-M}(\mathbf{d}-\mathbf{G m})^{T} \mathbf{C}_{d}^{-1}(\mathbf{d}-\mathbf{G m})
$$

where $\mathrm{N}$ is the number of data, $\mathrm{M}$ is the trace of the resolution matrix, $\mathbf{d}$ is the data vector $\left(d c_{1}(T)\right.$ or $d c_{2}(T)$ in equation 1$), \mathbf{m}$ represents the model parameters, $\mathbf{G}$ is the kernel matrix, and $\mathbf{C}_{d}$ is the data covariance matrix. We calculated the reduced $\chi^{2}$ misfit and trace of the resolution matrix at each grid cell for our initial $G_{c}$ and our initial $G_{s}$ models, as well as for $G_{s}$ and $G_{c}$ of model 1 and model 2. For each grid cell we then used a F-test to calculate the probability that YB13 and model 1 are identical and the probability that model YB13 and model 2 are identical. If this probability is low, the misfit reduction is significant and the model with the most parameters is relevant. This procedure resulted in probability maps for $G_{c}$ (Figs. 6(a) and 6(c)) and for $G_{s}$ (Figs. 6(b) and 6(d)). Figs. 6(a) and 6(b), which compare model YB13 and model 1, show that the probability that the two models are equivalent is less than $1 \%$ at most locations for both $G_{c}$ and $G_{s}$. The 
probability that the data require $G_{c}$ or $G_{s}$ anisotropy below $410 \mathrm{~km}$ is thus larger than 99\%. Figs.6(d) and 6(e), which compare YB13 and model 2 show that fewer locations are likely to require $G_{c}$ and $G_{s}$ to extend below $670 \mathrm{~km}$ depth, probably due to fact that only a few modes among the data used are sensitive to these depths.

However, because the anisotropy amplitude $\mathrm{G}$ is obtained by combining $G_{s}$ and $G_{c}$ (equation 6), the relevant information to determine whether deep upper mantle or lower anisotropy is needed is contained in panels (c) and (f), which represent the combined results for the two parameters. It is important to note that Figs. 6(c) and 6(f) are not probability maps, but just a vizualisation of where deep anisotropy is likely present. From a mathematical viewpoint, if events $\mathrm{A}$ and $\mathrm{B}$ represent the need for deep anisotropy in $G_{c}$ and $G_{s}$, respectively, we would need to calculate the probability of having A or $\mathrm{B}$, based on the probability map for $\mathrm{A}$ and the probability map for $\mathrm{B}$ in order to combine the results for $G_{c}$ and $G_{s}$. However, because events A and $\mathrm{B}$ are not mutually exclusive, combining them is not straightforward. We thus decided instead to construct Figs. 6(c) and 6(f) simply by associating a blue color to locations where both $G_{c}$ and $G_{s}$ have a probability larger than $5 \%$ that the models are equivalent, and a white color where either $G_{c}$ or $G_{s}$ has a low probability that the models are equivalent. Fig. 6(c) thus demonstrates that at least one of the two elastic parameters $\left(G_{c}\right.$ or $\left.G_{s}\right)$ needs to extend below $410 \mathrm{~km}$ depth at almost all latitudes and longitudes, i.e. that global MTZ seismic anisotropy is required by the data. Fig. 6(f)) shows that when combining the $G_{c}$ and $G_{s}$ F-test results, we find many grid cells where at least one of the two parameters is likely to be non-zero below the $670 \mathrm{~km}$ 
depth, even though many locations do not require deep $G_{c}$ or $G_{s}$ anisotropy.

Instead of comparing the $G_{c}$ models and the $G_{s}$ models at every grid cell separately, a more compact description of the model misfit can be obtained with an average $\chi^{2}$ :

$$
\bar{\chi}^{2}=\sum_{i=1}^{N_{p}}\left(\chi_{s, i}^{2}+\chi_{c, i}^{2}\right) / 2 N_{p}
$$

where $N_{p}$ is the number of grid cells, and $\chi_{s, i}^{2}$ and $\chi_{c, i}^{2}$ are the reduced $\chi^{2}$ for $G_{s}$ and $G_{c}$ at grid cell $i$, respectively. F-tests performed using these average misfits show that the probability of having anisotropy below $410 \mathrm{~km}$ is greater than $99 \%$, and that the probability of having anisotropy below $670 \mathrm{~km}$ is greater than $10 \%$ (Supplementary Fig. 15).

\subsection{Data uncertainties and misfit estimates}

The $\chi^{2}$ used above strongly depends on data uncertainties. However, despite Visser et al. (2008a)'s efforts to quantify phase velocity uncertainties, these are likely not fully characterized, which may affect the F-tests. For instance, theoretical assumptions made to obtain the measurements may bias uncertainty estimates. In addition, the data used here are anisotropic phase velocity maps, which were constructed by inversion of path-averaged phase velocity measurements. Our data are thus Visser et al. (2008a)'s models and our phase velocity map uncertainties result from their posterior model covariance. Both are affected by the regularization they applied. Furthermore, the uncertainties they reported were averaged over the sphere. They vary with the period and overtone number, but they do not change laterally and therefore do not account for the varying ray coverage quality across the globe. 
The variance reduction (VR) is another useful measure of data fit and is independent of data uncertainties. We thus calculated the VR averaged for all grid cells and for $G_{c}$ and $G_{s}$, and compared the values obtained for model YB13 with those obtained for the two models described in section 4.4. We also determined the VR for each overtone separately. Those values are reported in Table 1, and show that while our model explains over $85 \%$ of the data, model 1 and model 2 have a VR of only $74 \%$ and $80 \%$, respectively. The VR is also much higher for YB13 than for model 1 in each individual data subset. The VR of model 2 is closer to that of YB13, but lower for each data subset.

\subsection{Comparison with other models}

A quantitative comparison with anisotropy model DPK2005 (Debayle et al., 2005) is shown in Supplementary Fig. 20. We expanded DPK2005 in generalized spherical harmonics and calculated the correlation between the two models up to degree and order 20. This showed that the two models agree with one another above the $95 \%$ significance level in the top $275 \mathrm{~km}$. Discrepancies at larger depths are likely due to the different resolving powers of the data since the higher modes used in the present study significantly increased our sensitivity to deep upper mantle structure (Fig. 1) compared to the study of Debayle et al. (2005) who employed fundamental modes only.

Stronger differences can be found between our model and regional models such as that of Marone and Romanowicz (2007b) for North America. However, while the observed discrepancies can be partially attributed to the dif-

ferent datasets, inversion schemes, and regularization applied, another likely contributor is the difference in lateral resolution since our model anisotropy 
model has an estimated lateral resolution that is too low to resolve variations within North America.

Large discrepancies are also found between our model and the MTZ model of Trampert and van Heijst (2002). They might be due to different depth resolutions as this earlier work used a smaller dataset than ours, and it was composed of phase velocities for the first two Love wave overtones, which are significantly less sensitive to $V_{S V}$ anisotropy than Rayleigh waves. In addition, uncertainties on Love wave $2 \Psi$ anisotropy are typically larger than uncertainties on Rayleigh waves (Visser et al., 2008a), and the separation between fundamental and higher modes is more difficult for Love waves. Finally, Love wave $2 \Psi$ terms might also be biased due to Love-Rayleigh coupling as coupling exists in fundamental modes (Sieminski et al., 2007) and cannot be excluded yet for higher modes (Beghein et al., 2008).

\subsection{Shear-wave splitting predictions}

To verify that our model predicts reasonable shear-wave splitting delay times and fast azimuths we calculated splitting predictions following Montagner and Griot-Pommera (2000). Fig. 7 shows a qualitative overall agreement between predictions and station-averaged measurements. Comparing these two quantities is however not straightforward as they have very different spatial resolutions, and splitting measurements at a given station can differ between studies. Another complication is that our model is global whereas SKS splitting measurements are only available at seismic stations. A quantitative comparison of data and predictions can nevertheless be made using a generalized spherical harmonics expansion of the SKS data. We used the expansion of Becker et al. (2012) up to degree and order 20 (shown in blue 
in Fig. 7) and calculated a global correlation of 0.15, which is above the $95 \%$ confidence level for a degree 20 expansion.

One long-standing issue when comparing measurements of shear-wave splitting delay times and predictions based on surface wave tomographic models is that the predicted delay times are largely underestimated. Becker et al. (2012) showed that the global distribution of measured station-averaged delay times peaks around $1.1 \mathrm{~s}$ whereas delay times predicted by 3-D azimuthal anisotropy models peak around $0.3 \mathrm{~s}$. The authors determined a median delay time of $0.4 \mathrm{~s}$ for model DPK2005 using the method of Montagner and Griot-Pommera (2000) calculate the predictions. Fig. 8 shows however that our delay time predictions are closer to the observed values with a median around $0.75 \mathrm{~s}$. This demonstrates that we can better explain shear-wave splitting data with models that include deep upper mantle seismic anisotropy.

\section{Discussion}

In the upper $200-300 \mathrm{~km}$ of the mantle, our 3-D anisotropy pattern is in agreement with previous global studies (Nishimura and Forsyth, 1989; Montagner and Tanimoto, 1991; Debayle et al., 2005), and predicts shear-wave splitting values that are compatible with measurements. If we define the lithosphere and asthenosphere based on the goodness of alignment of $\Theta$ with the present-day APM (Fig.4), we can associate the transition between poor and good alignment with the lithosphere-asthenosphere boundary (LAB). We thus have a lithosphere of about $200 \mathrm{~km}$ thickness beneath cratons, which is compatible with a previous anisotropy-based estimate of continental roots 
thickness for North America (Yuan and Romanowicz, 2010). The fast azimuth becomes sub-parallel to the APM below that depth down to about $350 \mathrm{~km}$ depth, marking the bottom of a layer that we associate with the asthenosphere. Beneath oceans, the alignment of the fast axes with the APM occurs over depth ranges that vary with the age of the ocean floor, as expected from a lithosphere that thickens as it cools down and spreads away from the ridge. In the uppermost mantle our model can thus be interpreted in terms of olivine LPO in response to horizontal shear deformation due to convection in an asthenosphere of varying thickness, depending on the tectonic setting. The anisotropy signal found in the lithosphere ("frozen-in anisotropy") is generally interpreted in terms of past deformation.

Averaged over all longitudes and latitudes, the transition between poor and good alignment with the APM occurs around $80 \mathrm{~km}$ depth (Fig.4), which is also the depth at which we found a maximum in $d \Theta / d r$ (Fig.2). Similarly, the average depth at which the alignment with the APM breaks down and which might mark the bottom of the asthenosphere is around $250 \mathrm{~km}$ (Fig.4), which is also approximately the location of a maximum in $d \Theta / d r$. We thus interpret the high $d \Theta / d r$ and low G/L amplitude at $80 \mathrm{~km}$ depth in Fig.2 as the global signature of the LAB, and we speculate that the high $d \Theta / d r$ and low anisotropy amplitude found at about 230-250 km depth correspond to the Lehmann discontinuity (Fig.9). This discontinuity in seismic wave velocity is a major feature of PREM, but is generally not observed under young oceans (e.g., Deuss and Woodhouse (2002)).

For many years, the lack of evidence for seismic anisotropy below $\sim 250 \mathrm{~km}$ depth was interpreted in terms of deformation by diffusion creep, in which 
case LPO cannot develop (Karato, 1992). Our model demonstrates however that seismic anisotropy is present at least down to the bottom of the MTZ, thereby challenging existing paradigms regarding mantle deformation mechanisms. Olivine LPO could be responsible for the anisotropy detected between $250 \mathrm{~km}$ and $410 \mathrm{~km}$ depth. High-pressure experiments (Mainprice et al., 2005) suggest indeed that dislocation creep dominate in the deep upper mantle, with a low resulting LPO anisotropy $(<2 \%)$ that is compatible with our model amplitudes. Deformation at these depths could occur with a different slip system than at shallower depths (Mainprice et al., 2005), which implies that the anisotropy fast axis may not be a good proxy for flow direction in this part of the mantle, contrary to what is generally observed closer to the surface. The change in seismic fast direction found around $250 \mathrm{~km}$ depth may thus not reflect a change in shear direction (Fig. 9(b) and (c)).

The newly detected correlation between reduced anisotropy amplitude, changes in fast azimuth of propagation, and the location of upper mantle phase transitions is of particular interest, and strongly suggests that the LPO of MTZ material is at the origin of the anisotropy. LPO of wadsleyite and/or ringwoodite could indeed explain the observed signal in the upper and lower transition zone, respectively, as they have been shown to be intrinsically anisotropic. Wadsleyite single crystal anisotropy is about $14 \%$ (Zha et al., 1997), and recent modeling showed that a polycrystal of pyrolytic composition at MTZ conditions can have 1\% seismic anisotropy (Tommasi et al., 2004; Kawazoe et al., 2013), compatible with our model. The intrinsic elastic anisotropy of ringwoodite is more ambiguous. It is often believed to have no intrinsic anisotropy (Kiefer et al., 1997), but some laboratory 
experiments showed that both anhydrous and hydrous ringwoodite can be anisotropic (Kavner, 2003). It was also proposed that the detection of MTZ anisotropy could be the signature of volatiles as their presence increases the ductile strain rates of olivine aggregates, resulting in stronger LPO and thus stronger seismic anisotropy (Kavner, 2003). LPO of ringwoodite is thus a possible explanation for the observed global anisotropy in the lower MTZ. In the top of the lower mantle, candidates for LPO are magnesiowustite (Karki et al., 1997) and perovskite (Karato, 1998).

Recrystallization or a change in the slip system of the olivine structure during phase changes could explain both the high gradients in fast direction and the amplitude lows at $410 \mathrm{~km}$ and $670 \mathrm{~km}$ depth since the anisotropy would be erased during the transitions before building up again. If water is present at these depths (Bercovici and Karato, 2003) it might indeed have a similar effect on the anisotropy of MTZ material as it has on olivine anisotropy at shallower depths (Jung and Karato, 2001). A change in water content owing to differences in olivine and MTZ minerals solubility (Kohlstedt et al., 1996) could then result in a change in the slip system across MTZ boundaries.

Inferring the style of mantle convection from our model is not straightforward owing to the lack of constraints on MTZ anisotropy from mineral physics and to the large uncertainties on radial anisotropy at these depths. According to mineral physics analyses by Tommasi et al. (2004) horizontal shear has to be dominant in the MTZ in order to be compatible with radial anisotropy models showing $V_{S H}>V_{S V}$ (Montagner and Kennett, 1996; Beghein et al., 2006; Panning and Romanowicz, 2006) and with $V_{S V}$ az- 
imuthal anisotropy stronger than $V_{S H}$ anisotropy as found by Trampert and van Heijst (2002). Models of radial anisotropy have however large uncertainties at these depths (Beghein and Trampert, 2004b; Beghein et al., 2006) and other results show $V_{S H}<V_{S V}$ in the MTZ (Visser et al., 2008b). Furthermore, a recent mineral physics study argued that wadsleyite aggregates deform with a fast direction for $V_{S V}$ that is perpendicular to the shear plane, i.e. with $V_{S V}>V_{S H}$ in horizontal flow and with $V_{S H}>V_{S V}$ in vertical flow (Kawazoe et al., 2013). Based on results by Visser et al. (2008b) this would translate into horizontal shear, but using other models (Montagner and Kennett, 1996; Beghein et al., 2006; Panning and Romanowicz, 2006) this would result in vertical shear instead.

Keeping these caveats in mind, if we assume that vertically polarized shear-wave fast directions directly reflect mantle flow direction at all depths (i.e., assuming horizontal shear) the global changes in anisotropy amplitudes and directions around $250 \mathrm{~km}$ depth and across the MTZ discontinuities could be interpreted in terms of changes in horizontal flow direction, most compatible with layered convection models (Fig. 9a). However, seismic fast directions and mantle flow are not necessarily parallel below $\sim 250 \mathrm{~km}$ depth (Mainprice et al., 2005), and the relation between seismic and intrinsic crystal anisotropy in the MTZ is unknown. A scenario in which plate motion results from shear distributed over a broader layer than traditionally thought cannot therefore be ruled out (Fig. 9b and 9c), as previously suggested in a shear-wave splitting study (Kosarian et al., 2011). 


\section{Conclusions}

We modeled the 3-D variations in SV azimuthal anisotropy in the top $1000 \mathrm{~km}$ of the mantle using fundamental modes and overtone Rayleigh wave phase velocity maps (Visser et al., 2008a). These data have much higher sensitivity to deep upper mantle and lower mantle structure than previous datasets. We found a complex pattern of seismic anisotropy that agrees with previous models in the top $250 \mathrm{~km}$ of the mantle, but that displays multiple changes in the fast $V_{S V}$ azimuth with depth. A global change in fast azimuth associated with a low anisotropy amplitude was detected around $80 \mathrm{~km}$ and $230 \mathrm{~km}$ depth, which we attributed to the average signatures of the LAB and of the bottom of the asthenosphere, respectively. We also speculate that the bottom of the asthenosphere might correspond to the location of the Lehmann discontinuity. Most interestingly, we also found a high gradient in SV wave fast direction across the MTZ boundaries and a previously undetected correlation between high gradients in fast axes and a reduced seismic anisotropy amplitude.

Our 3-D model can be interpreted in terms of olivine LPO in the upper mantle associated to horizontal shear deformation down to at least the bottom of the asthenosphere. At larger depths, the interpretation of our results is more difficult because it is highly dependent on mineral physics analyses and on radial anisotropy models, which are both associated with large uncertainties. While we cannot distinguish between layered and whole mantle convection at this stage, our model provides unique new constraints on mantle deformation. Advances toward a better understanding of Earth's convective pattern will not only require stronger constraints on radial anisotropy 
models, but the effects of pressure, melting, water content, and deformation mechanisms and slip systems of MTZ materials will need to be addressed by mineral physicists.

\section{Acknowledgements}

This research was funded by NSF EAR grant numbers 0838605 and 0949255. The authors wish to thank Jeannot Trampert and Karin Visser for freely sharing their phase velocity maps, Eric Debayle and Jeroen Ritsema for making their models available online, and UCLA colleagues Paul Davis and David Jackson for fruitful discussions. Thorsten Becker provided the spherical harmonic expansion of the shear-wave splitting data. Two anonymous reviewers helped improve this manuscript. Partial derivatives were calculated using computer program MINEOS (available on the CIG website at http://www.geodynamics.org/), and figures were made using the Generic Mapping Tool, Gnuplot, and Xmgrace.

\section{References}

Anderson, D. L., 1967. Phase changes in the upper mantle. Science 157 (3793), 1165-1166.

Bassin, C., Laske, G., Masters, G., 2000. The current limits of resolution for surface wave tomography in north america. In: EOS Trans AGU. Vol. 81.

Becker, T. W., Lebedev, S., Long, M. D., 2012. On the relationship between azimuthal anisotropy from shear wave splitting and surface wave tomography. Journal of Geophysical Research 117 (B1). 
Beghein, C., 2010. Radial anisotropy and prior petrological constraints: A comparative study. Journal of Geophysical Research 115 (B3), 1-17.

Beghein, C., Resovsky, J., van der Hilst, R. D., 2008. The signal of mantle anisotropy in the coupling of normal modes. Geophysical Journal International 175 (3), 1209-1234.

Beghein, C., Trampert, J., 2004a. Probability density functions for radial anisotropy from fundamental mode surface wave data and the Neighbourhood Algorithm. Geophysical Journal International 157 (3), 1163-1174.

Beghein, C., Trampert, J., 2004b. Probability density functions for radial anisotropy: implications for the upper $1200 \mathrm{~km}$ of the mantle. Earth and Planetary Science Letters 217 (1-2), 151-162.

Beghein, C., Trampert, J., van Heijst, H.-J., 2006. Radial anisotropy in seismic reference models of the mantle. Journal of Geophysical Research $111(\mathrm{~B} 2), 1-9$.

Bercovici, D., Karato, S., 2003. Whole-mantle convection and the transitionzone water filter. Nature 425, 39-44.

Bevington, P. R., Robinson, D. K., 2002. Data reduction and error analysis for the physical sciences, 3rd Edition. Vol. 336. McGraw-Hill.

Boschi, L., Ekström, G., 2002. New images of the Earth's upper mantle from measurements of surface wave phase velocity anomalies. Journal Of Geophysical Research-Solid Earth 107 (B4), 2059. 
Bozdağ, E., Trampert, J., 2010. Assessment of tomographic mantle models using spectral element seismograms. Geophysical Journal International $180(3), 1187-1199$.

Debayle, E., Kennett, B., Priestley, K., 2005. Global azimuthal seismic anisotropy and the unique plate-motion deformation of Australia. Nature 433 (7025), 509-512.

Deuss, A., Woodhouse, J. H., 2002. A systematic search for mantle discontinuities using SS-precursors. Geophysical Research Letters 29 (8), 90 1-4.

Dziewonski, A. M., Anderson, D. L., 1981. Preliminary reference Earth model. Physics of the Earth and Planetary Interiors 25 (4), 297-356.

Ferreira, A., Woodhouse, J. H., Visser, K., Trampert, J., 2010. On the robustness of global radially anisotropic surface wave tomography. Journal of Geophysical Research - Solid Earth 115 (B04313).

Fouch, M. J., Fischer, K. M., 1996. Mantle anisotropy beneath northwest Pacific subduction zones. Journal Of Geophysical Research 101 (B7), 1598716002.

Gripp, A. E., Gordon, R. G., 2002. Young tracks of hotspots and current plate velocities. Geophysical Journal International 150 (2), 321-361.

Hager, B. H., O'Connell, R. J., 1979. Kinematic Models of Large-Scale Flow in the Earth's Mantle. Journal of Geophysical Research 84 (B3), 10311048. 
Jung, H., Karato, S., 2001. Water-induced fabric transitions in olivine. Science 293 (5534), 1460-1463.

Karato, S., 1992. On the Lehmann Discontinuity. Geophysical Research Letters 19 (22), 2255-2258.

Karato, S., 1998. Seismic anisotropy in the deep mantle, boundary layers and the geometry of mantle convection. Pure and Applied Geophysics 151 (24), 565-587.

Karato, S., Wu, P., 1993. Rheology of the upper mantle: A synthesis. Science 260 (5109), 771-778.

Karki, B., Stixrude, L., Clark, S., Warren, M., Ackland, G. J., Crain, J., 1997. Structure and elasticity of $\mathrm{MgO}$ at high pressure. American Mineralogist $82(1-2), 51-60$.

Kavner, A., 2003. Elasticity and strength of hydrous ringwoodite at high pressure. Earth and Planetary Science Letters 214 (3-4), 645-654.

Kawazoe, T., Ohuchi, T., Nishihara, Y., Nishiyama, N., Fujino, K., Irifune, T., 2013. Seismic anisotropy in the mantle transition zone induced by shear deformation of wadsleyite. Physics of the Earth and Planetary Interiors 216, 91-98.

Kiefer, B., Stixrude, L., Wentzcovitch, R. M., 1997. Calculated elastic constants and anisotropy of $\mathrm{Mg}_{2} \mathrm{SiO}_{4}$ spinel at high pressure. Geophysical Research Letters 24 (22), 2841-2844. 
Kohlstedt, D. L., Keppler, H., Rubie, D. C., 1996. Solubility of water in the alpha, beta, and gamma phases of $(\mathrm{Mg}, \mathrm{Fe})_{2} \mathrm{SiO}_{4}$. Contributions to Mineralogy and Petrology 123 (4), 345-357.

Kosarian, M., Davis, P. M., Tanimoto, T., Clayton, R. W., 2011. The relationship between upper mantle anisotropic structures beneath California, transpression, and absolute plate motions. Journal Of Geophysical Research 116 (B8).

Kustowski, B., Dziewonski, A., Ekström, G., 2007. Nonlinear crustal corrections for normal-mode seismograms. Bulletin of the Seismological Society of America 97 (5), 1756-1762.

Kustowski, B., Ekström, G., Dziewonski, A., 2008. Anisotropic shear-wave velocity structure of the Earth's mantle: A global model. Journal Of Geophysical Research-Solid Earth 113 (B6), B06306.

Love, A. E. H., 1927. Theory of Elasticity. Cambridge.

Mainprice, D., Tommasi, A., Couvy, H., Cordier, P., Frost, D. J., 2005. Pressure sensitivity of olivine slip systems and seismic anisotropy of Earth's upper mantle. Nature 433, 731-733.

Marone, F., Romanowicz, B., 2007a. Non-linear crustal corrections in highresolution regional waveform seismic tomography. Geophysical Journal International $170(1), 460-467$.

Marone, F., Romanowicz, B., 2007b. The depth distribution of azimuthal anisotropy in the continental upper mantle. Nature 447, 198-U4. 
Matsu'ura, M., Hirata, N., 1982. Generalized least-squares solutions to quasilinear inverse problems with a priori information. Journal of Physics of the Earth 30 (6), 451-468.

Montagner, J.-P., Griot-Pommera, D., 2000. How to relate body wave and surface wave anisotropy? Journal of Geophysical Research - Solid Earth 105 (B8), 15-19,19,27.

Montagner, J.-P., Kennett, B., 1996. How to reconcile body-wave and normal-mode reference earth models. Geophysical Journal International 125 (1), 229-248.

Montagner, J.-P., Nataf, H. C., 1986. A simple method for inverting the azimuthal anisotropy of surface waves. Journal of Geophysical Research Solid Earth 91 (B1), 511-520.

Montagner, J.-P., Tanimoto, T., 1991. Global upper mantle tomography of seismic velocities and anisotropies. Journal of Geophysical Research - Solid Earth 96 (B12), 20337-20351.

Nettles, M., Dziewonski, A., 2008. Radially anisotropic shear velocity structure of the upper mantle globally and beneath North America. Journal Of Geophysical Research-Solid Earth 113 (B2), B02303.

Nishimura, C. E., Forsyth, D. W., 1989. The anisotropic structure of the upper mantle in the Pacific. Geophysical Journal 96 (2), 203-229.

Panning, M., Romanowicz, B., 2006. A three-dimensional radially anisotropic model of shear velocity in the whole mantle. Geophysical Journal International 167, 361-379. 
Phinney, R. A., Burridge, R., 1973. Representation of Elastic-Gravitational Excitation of a Spherical Earth Model by Generalized Spherical Harmonics. Geophysical Journal Of The Royal Astronomical Society 34 (4), 451-487.

Schilling, J.-G., 1973. Iceland Mantle Plume: Geochemical Study of Reykjanes Ridge. Nature 242 (5400), 565-571.

Shearer, P., 2000. Upper mantle seismic discontinuities. In: Karato, S.-I., Forte, A., Liebermann, R., Masters, G., Stixrude, L. (Eds.), Earth's Deep Interior: Mineral Physics and Tomography From the Atomic to the Global Scale. Vol. 117. AGU, pp. 115-131.

Sieminski, A., Liu, Q., Trampert, J., Tromp, J., 2007. Finite-frequency sensitivity of surface waves to anisotropy based upon adjoint methods. Geophysical Journal International 168, 1153-1174.

Smith, M., Dahlen, F. A., 1973. The azimuthal dependence of Love and Rayleigh wave propagation in a slightly anisotropic medium. Journal of Geophysical Research - Solid Earth 78 (17), 3321-3333.

Takeuchi, H., Saito, M., 1972. Seismic surface waves. In: Bolt, B. A. (Ed.), Methods in Computational Physics. Academic Press, New York, pp. 217295.

Tommasi, A., Mainprice, D., Cordier, P., Thoraval, C., Couvy, H., 2004. Strain-induced seismic anisotropy of wadsleyite polycrystals and flow patterns in the mantle transition zone. Journal of Geophysical Research 109 (B21), B12405. 
Trampert, J., van Heijst, H. J., 2002. Global azimuthal anisotropy in the transition zone. Science 296, 1297-1299.

Trampert, J., Woodhouse, J. H., 2003. Global anisotropic phase velocity maps for fundamental mode surface waves between 40 and $150 \mathrm{~s}$. Geophysical Journal International 154, 154-165.

Visser, K., Trampert, J., Kennett, B. L. N., 2008a. Global anisotropic phase velocity maps for higher mode Love and Rayleigh waves. Geophysical Journal International 172, 1016-1032.

Visser, K., Trampert, J., Lebedev, S., Kennett, B. L. N., 2008b. Probability of radial anisotropy in the deep mantle. Earth and Planetary Science Letters 270, 241-250.

Yuan, H., Romanowicz, B., 2010. Lithospheric layering in the North American craton. Nature 466 (7310), 1063-1069.

Zha, C., Duffy, T., Mao, H., Downs, R., Hemley, R., Weidner, D., 1997. Single-crystal elasticity of $\beta-\mathrm{Mg}_{2} \mathrm{SiO}_{4}$ to the pressure of the $410 \mathrm{~km}$ seismic discontinuity in the Earth's mantle. Earth and Planetary Science Letters 147 (1-4), E9-E15. 
Figure 1: Rayleigh wave phase velocity partial derivatives calculated for perturbations in parameter $G$ with respect to Love (Love, 1927) parameter $L\left(L=\rho V_{S V}^{2}\right.$, where $\rho$ represents the density and $V_{S V}$ is the velocity of vertically polarized shear-waves) using PREM for the fundamental $(\mathrm{n}=0)$ and higher modes ( $\mathrm{n}=1$ through 6 ) employed in this study. We used 79 modes total, including 16 fundamental modes between 35 and 175 s, 16 first overtones between 35 and $172 \mathrm{~s}, 15$ second overtones between 35 and $149 \mathrm{~s}$, 11 third overtones between 35 and $88 \mathrm{~s}, 8$ fourth overtones between 35 and $62 \mathrm{~s}, 7$ fifth overtones between 35 and $56 \mathrm{~s}$, and 6 sixth overtones between 35 and $50 \mathrm{~s}$.

Figure 2: (a) Root mean square of relative azimuthal anisotropy amplitude with respect to elastic parameter L. DPK2005 represents the previously published model of Debayle et al. (2005); (b) Root mean square vertical gradient of the fast azimuth of propagation. The gradient was calculated for each grid cell as a function of depth with a $10 \mathrm{~km}$ spacing and a $20 \mathrm{~km}$ window, after which the rms gradient was determined as a function of depth.

Figure 3: Global 3-D azimuthal anisotropy model. The red bars represent the fast direction of propagation for vertically polarized shear-waves and their length is proportional to the amplitude of the anisotropy. The background grey scale is also indicative of the anisotropy relative amplitude. Plate boundaries are shown by thin black lines. The maximum anisotropy amplitude is displayed on top of each panel. 
Figure 4: Deviation of fast azimuth from the APM under stable continents and oceans of increasing ages. The global average is represented by the thin blue line.

Figure 5: Synthetic tests for a variety of input $G_{c} / L$ or $G_{s} / L$ models. In all panels the input model has $2 \%$ anisotropy in the top $200 \mathrm{~km}$ of the mantle. The input model of panel (a) has no anisotropy below that depth; Input models (b) and (c) include $1.5 \%$ anisotropy in the top and the bottom of the MTZ, respectively; Input model (d) includes $1.5 \%$ anisotropy across the entire MTZ; Input model (e) is identical to input model (d) but also includes $1 \%$ anisotropy in the top of the lower mantle, between $800 \mathrm{~km}$ and $900 \mathrm{~km}$ depth; Input model (f) has a constant amount of anisotropy through the top $800 \mathrm{~km}$ of the mantle

Figure 6: F-tests performed for $G_{c}((\mathrm{a})$ and $(\mathrm{d}))$ and $G_{s}((\mathrm{~b})$ and (e)) to compare our model with model 1 ((a), (b), and (c)) and with model 2 $((\mathrm{d}),(\mathrm{e})$, and (f)), as described in the main text. White areas in (a) and (b) correspond to places in which the likelihood that anisotropy is not needed below $410 \mathrm{~km}$ depth is at most $1 \%$ for $G_{c}$ and $G_{s}$, respectively. White areas in (d) and (e) show where the likelihood that anisotropy is not needed below $670 \mathrm{~km}$ depth is less or equal to $1 \%$ for $G_{c}$ and $G_{s}$, respectively. Light grey areas are associated with a 1-5\% probability. Panels (c) and (f) represent a combination of the $G_{c}$ and for $G_{s}$ F-tests, but do not correspond to a probability map (see main text). The white color in (c) therefore shows where anisotropy is likely to be located below $410 \mathrm{~km}$ depth, and white color 
in panel (f) denotes where anisotropy is likely to be located below $670 \mathrm{~km}$ depth.

Figure 7: Comparison between the station-averaged shear-wave splitting data, the degree 20 generalized spherical harmonic expansion of Becker et al. (2012) of the (not-averaged) data, and the model predictions. The data are shown by the grey sticks, the spherical harmonic expansion is in blue, and the model predictions are in red. The station-averaged data shown (http://geodynamics.usc.edu/ becker/) is an updated version of that of Becker et al. (2012).

Figure 8: Distribution of shear-wave splitting delay times from the stationaveraged database of Becker et al. (2012), compared to predictions based on model DPK2005 (Debayle et al., 2005) (top) and our model (bottom).

Figure 9: Illustration of three possible convection scenarios. The red curve represents the rms anisotropy amplitude. In all cases, frozen-in lithospheric anisotropy overlies anisotropy with fast axes sub-parallel to the APM in the asthenosphere, the thickness of which depends on the tectonic setting (Fig. 4). Fast anisotropy axes change direction below the asthenosphere and in the MTZ. We do not speculate on anisotropy in the lower mantle as our vertical resolution is lower below $700-800 \mathrm{~km}$ depth. The changes in the shaded patterns illustrate changes in anisotropy fast axes without necessarily representing the actual fast seismic direction. (a) Plate motion and anisotropy 
are caused by shear in the asthenosphere, and deeper anisotropy relates to a different mantle flow direction, decoupled from asthenospheric deformation. Below the asthenosphere, anisotropy fast axes may or may not align with flow direction. (b) Plate motion is caused by a broad shear zone that extends to $410 \mathrm{~km}$ depth. Anisotropy fast axes below the asthenosphere do not coincide with APM due to the effects of pressure, melting, water content, and/or deformation mechanisms (Mainprice et al., 2005). The MTZ is characterized by a different flow direction and different fast anisotropy axes that may or may not align with the flow direction; (c) Same as (b) but the shear zone spans at least the top $670 \mathrm{~km}$ of the mantle.

Table 1: Average variance reduction (VR) for our model (YB13), a model with no anisotropy below $410 \mathrm{~km}$ (model 1), and a model with no anisotropy below $670 \mathrm{~km}$ (model 2). These are the same models as those used for the F-test presented in the main manuscript. The VR was calculated for each data subset of overtone number $n$ and for all data together $(n=0-6)$. 


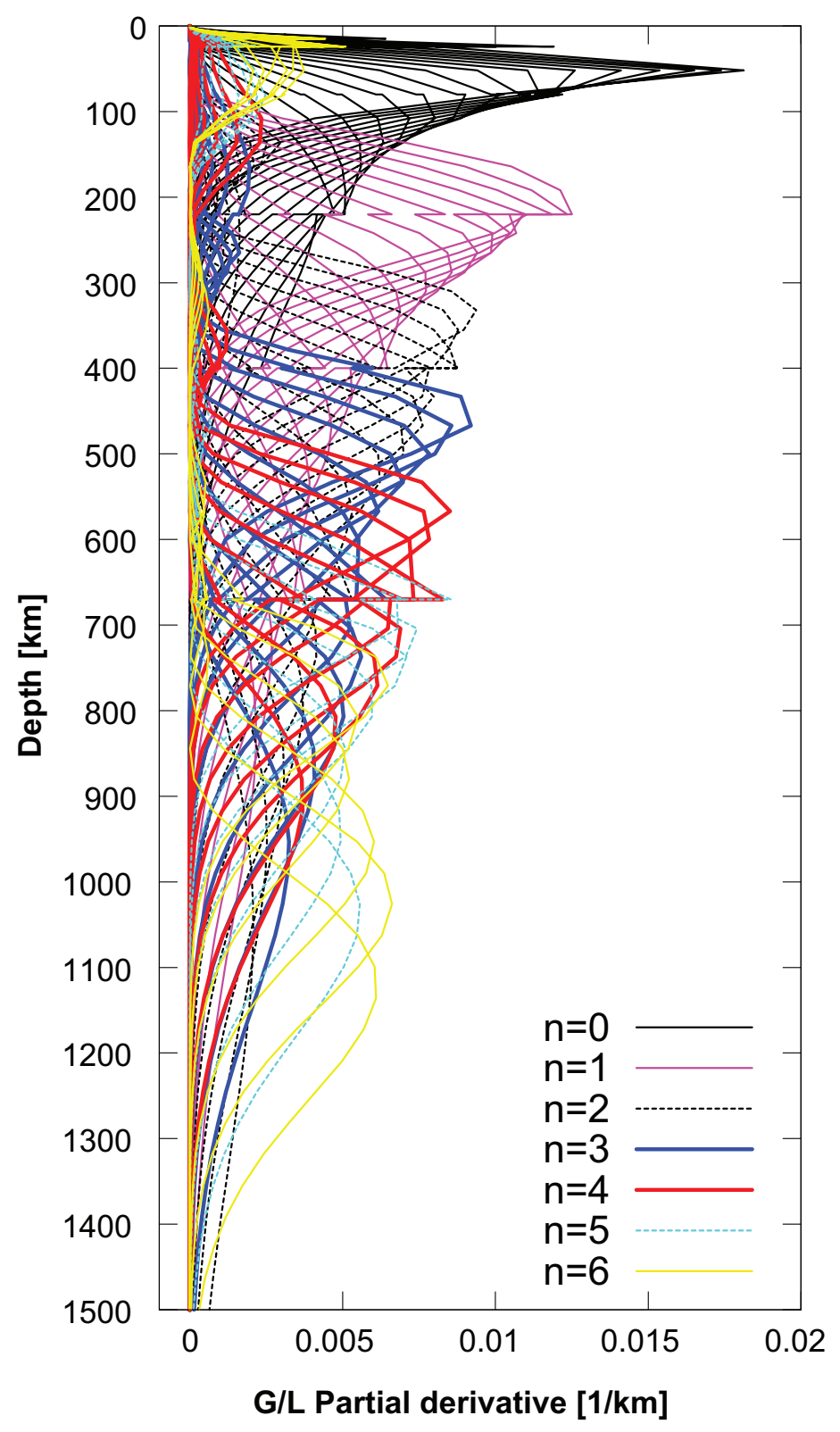

Figure 1: 


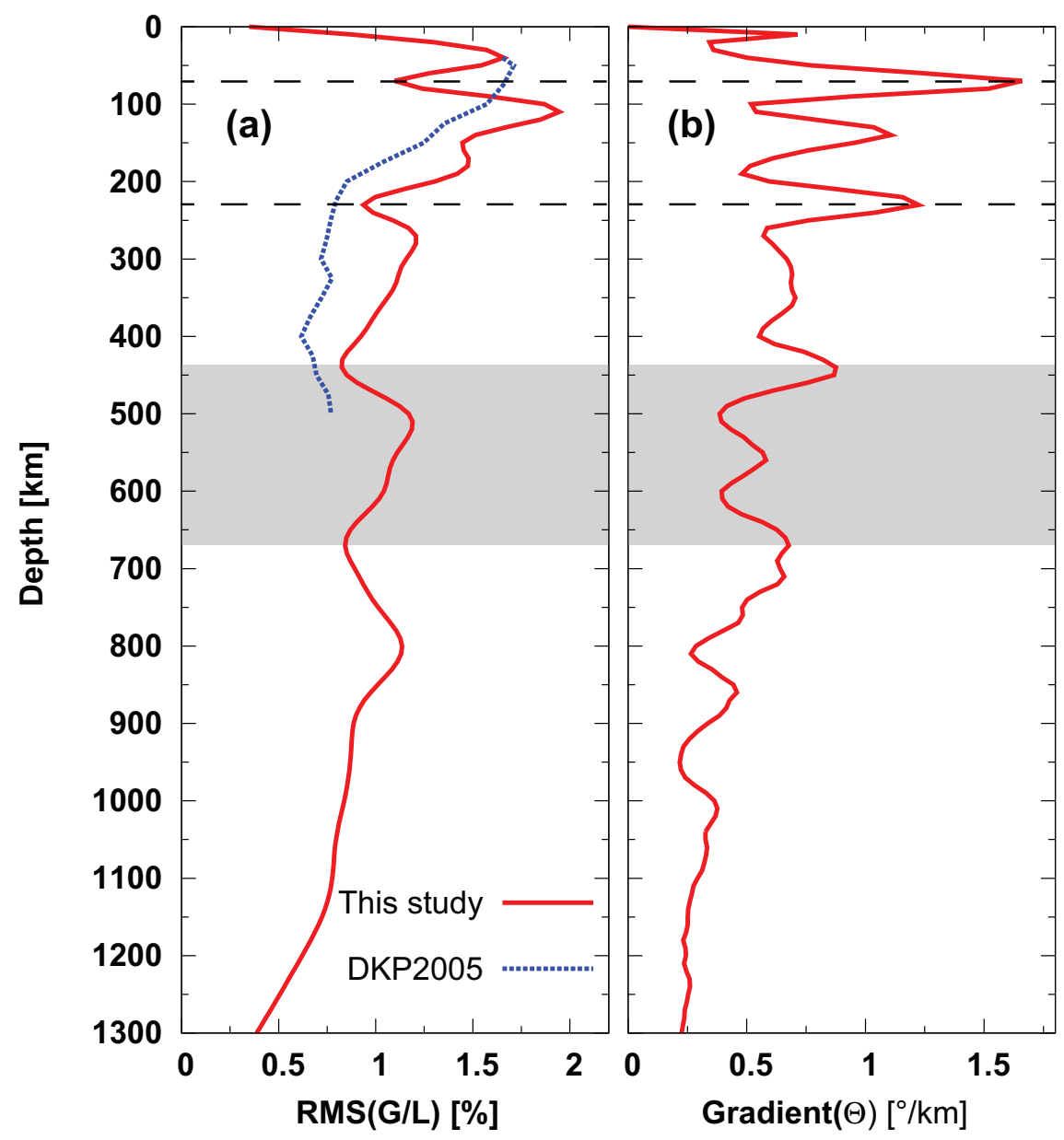

Figure 2: 

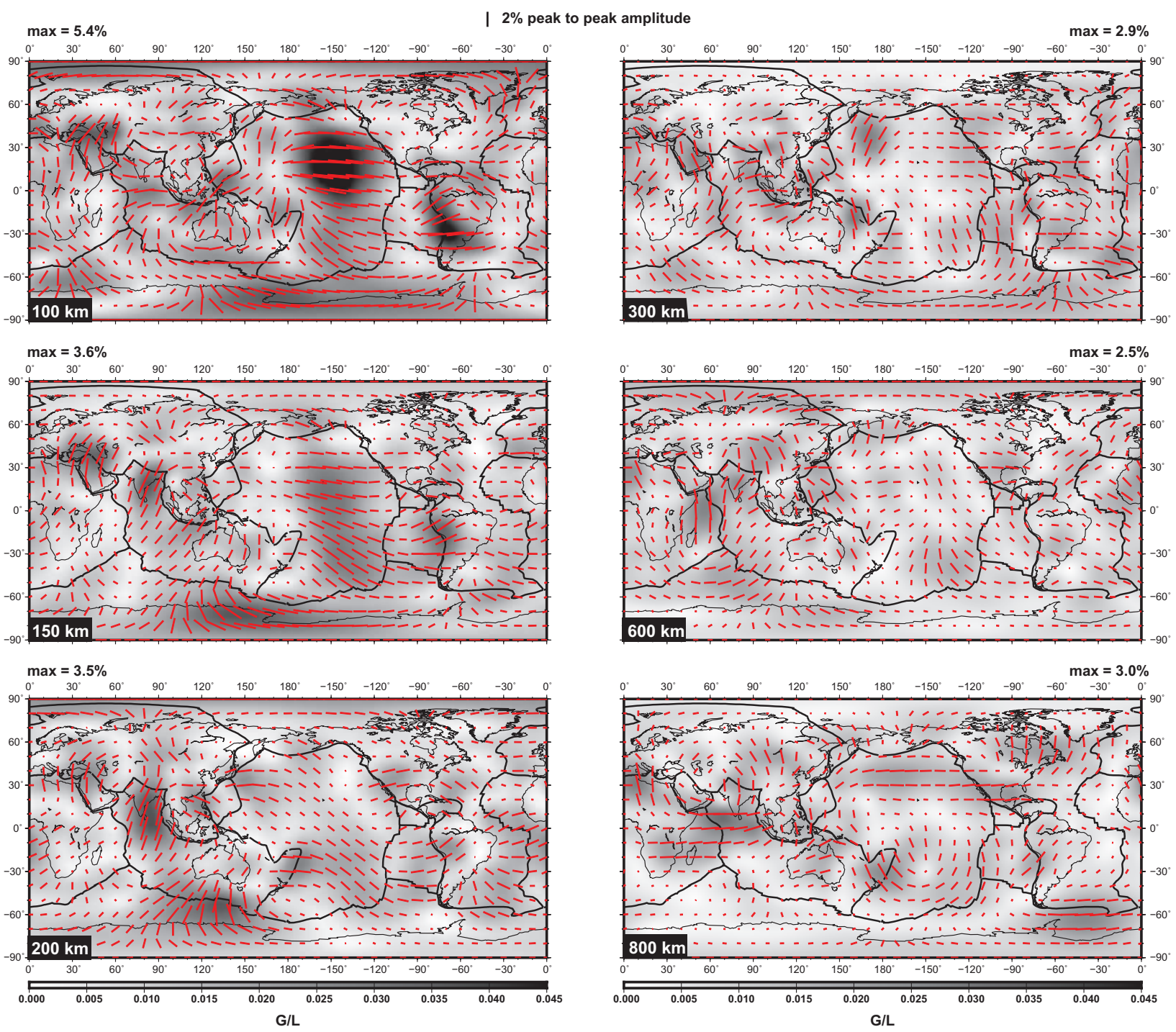

Figure 3: 


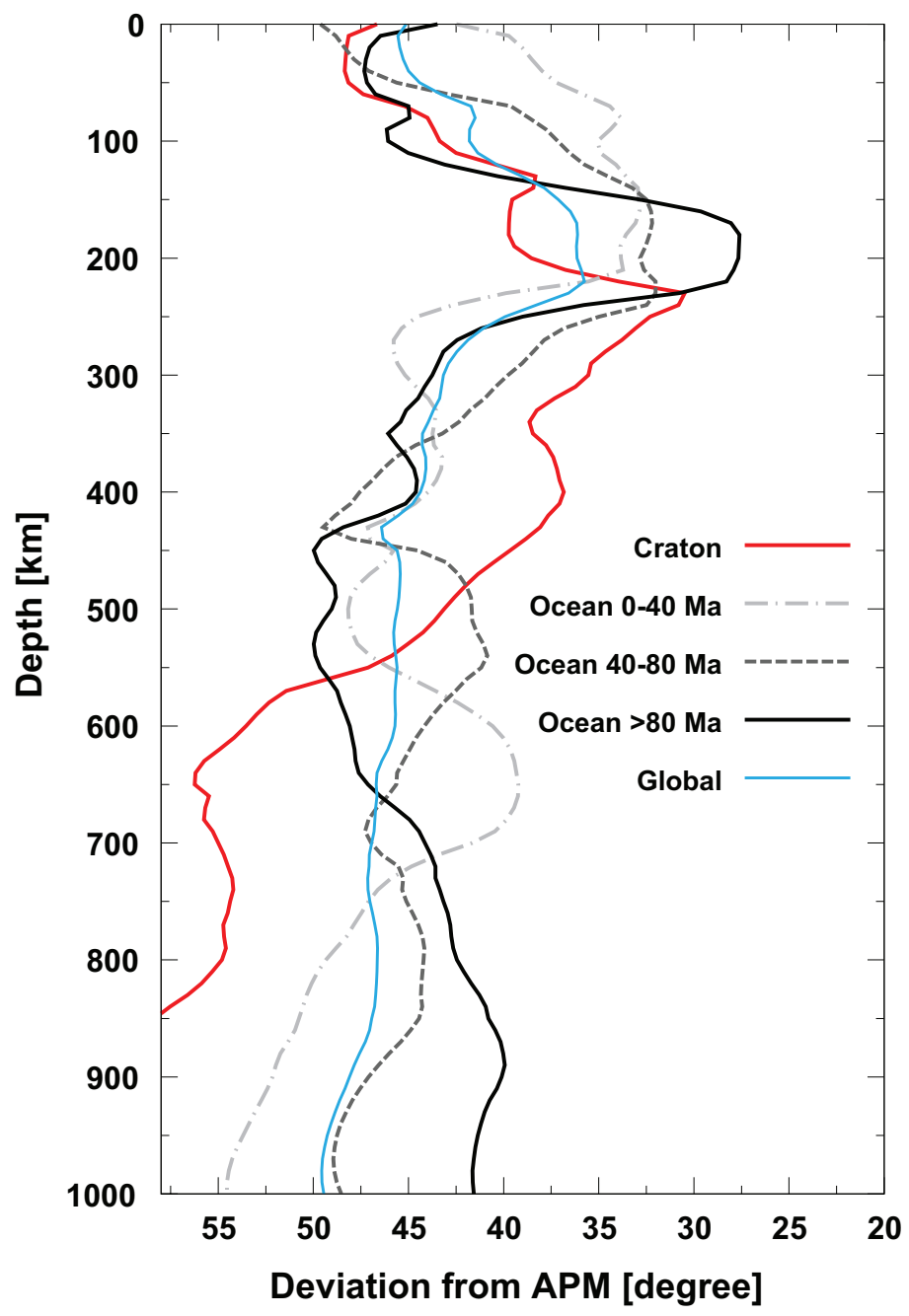

Figure 4: 

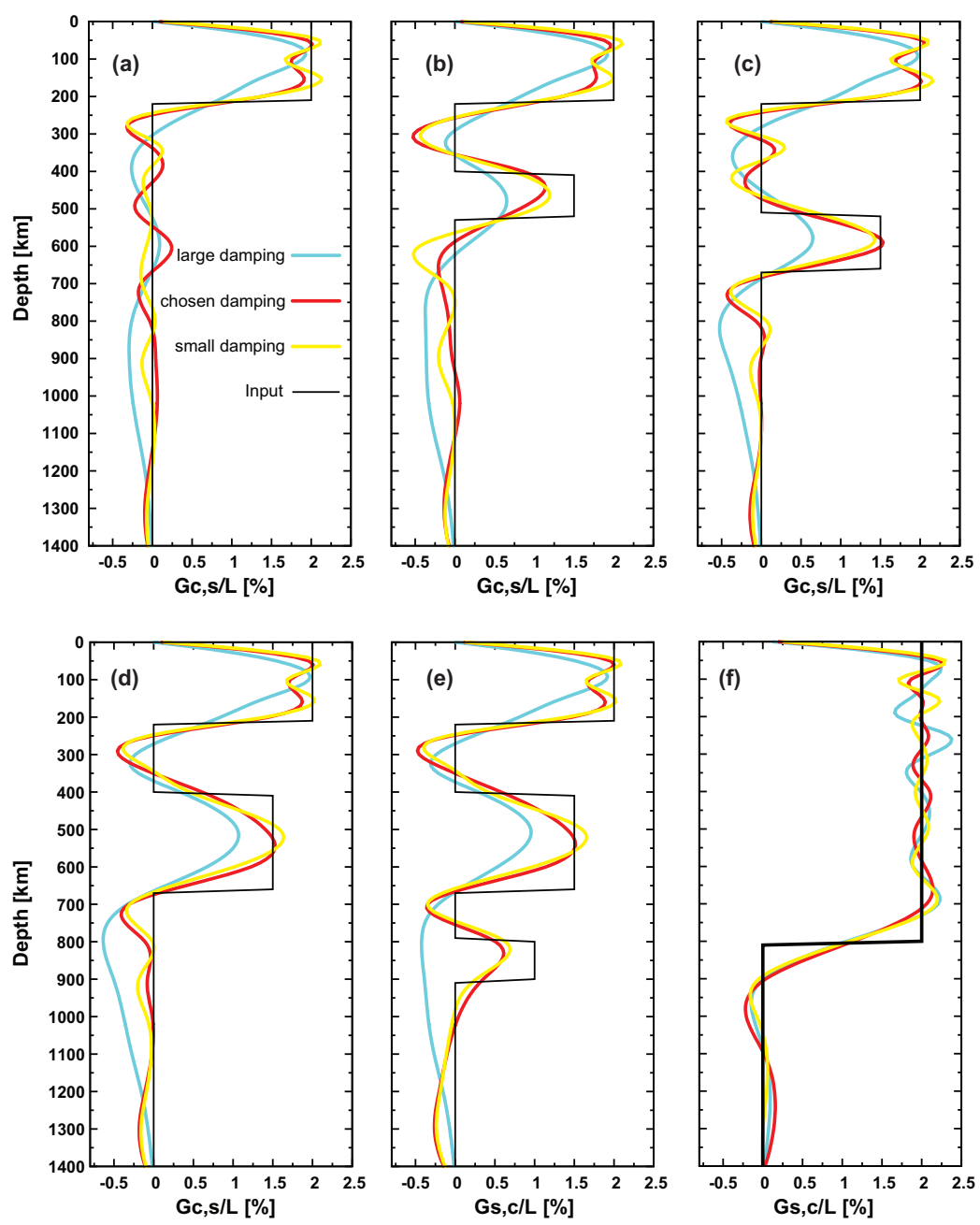

Figure 5: 

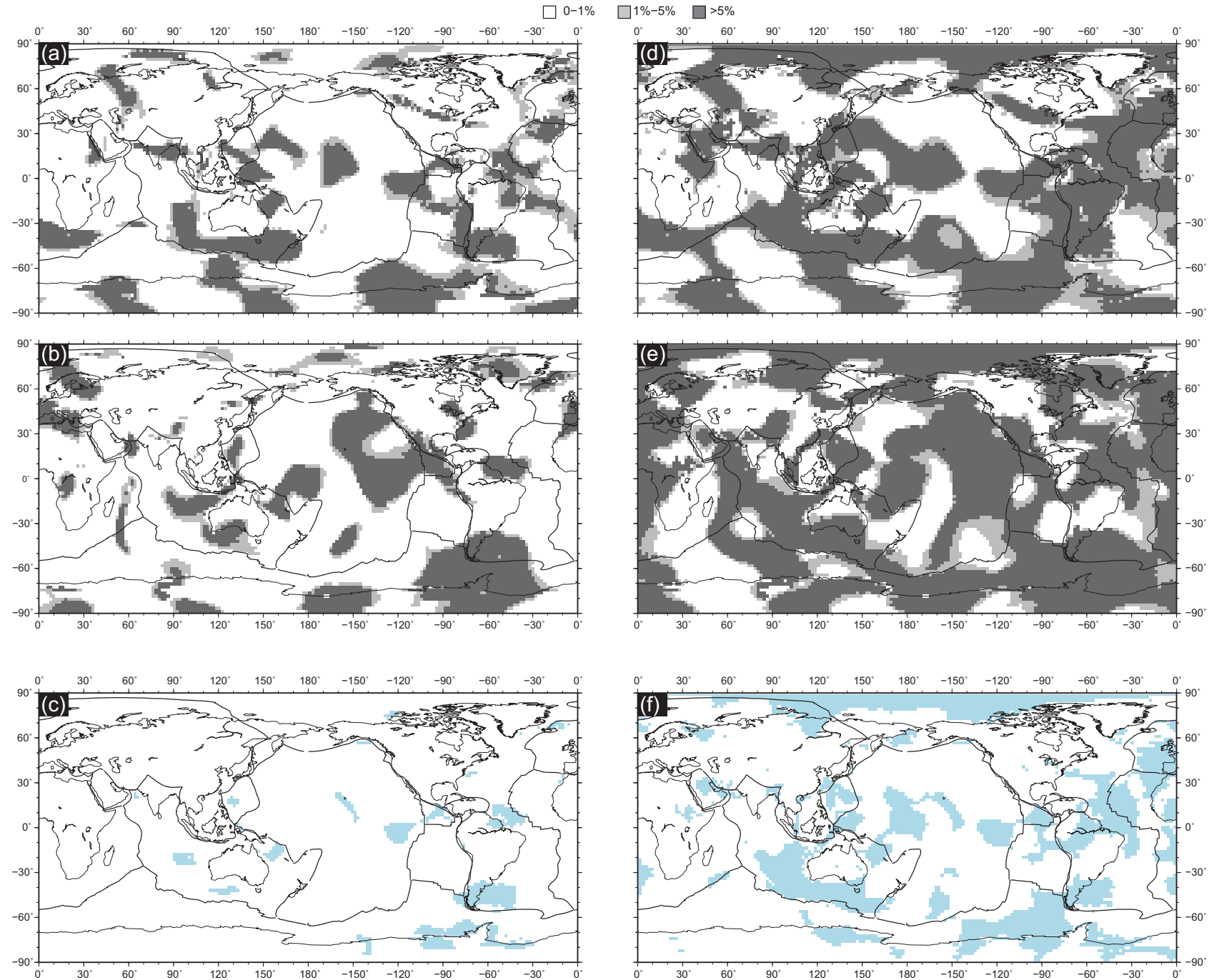

Figure 6: 


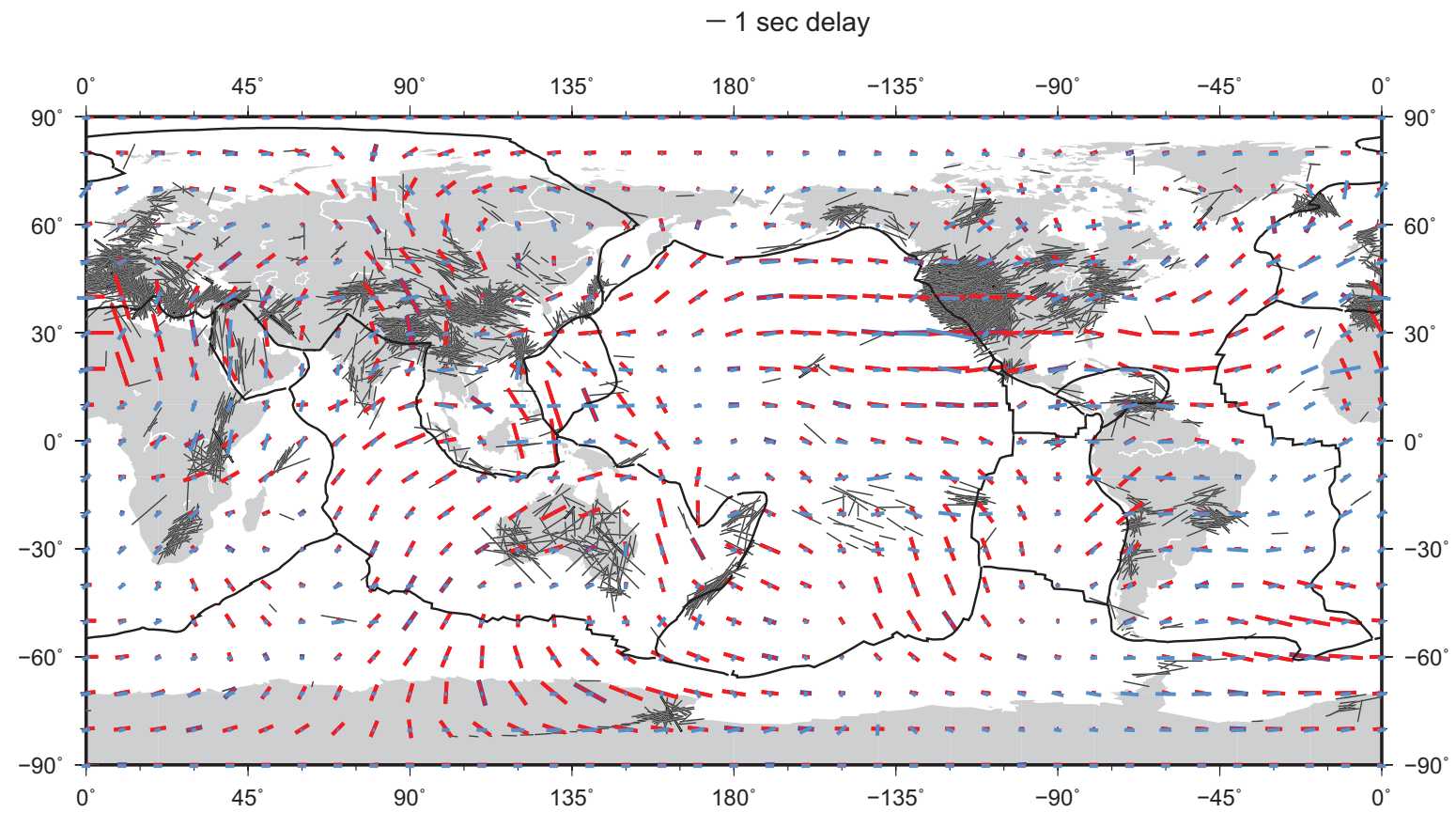

Figure 7: 

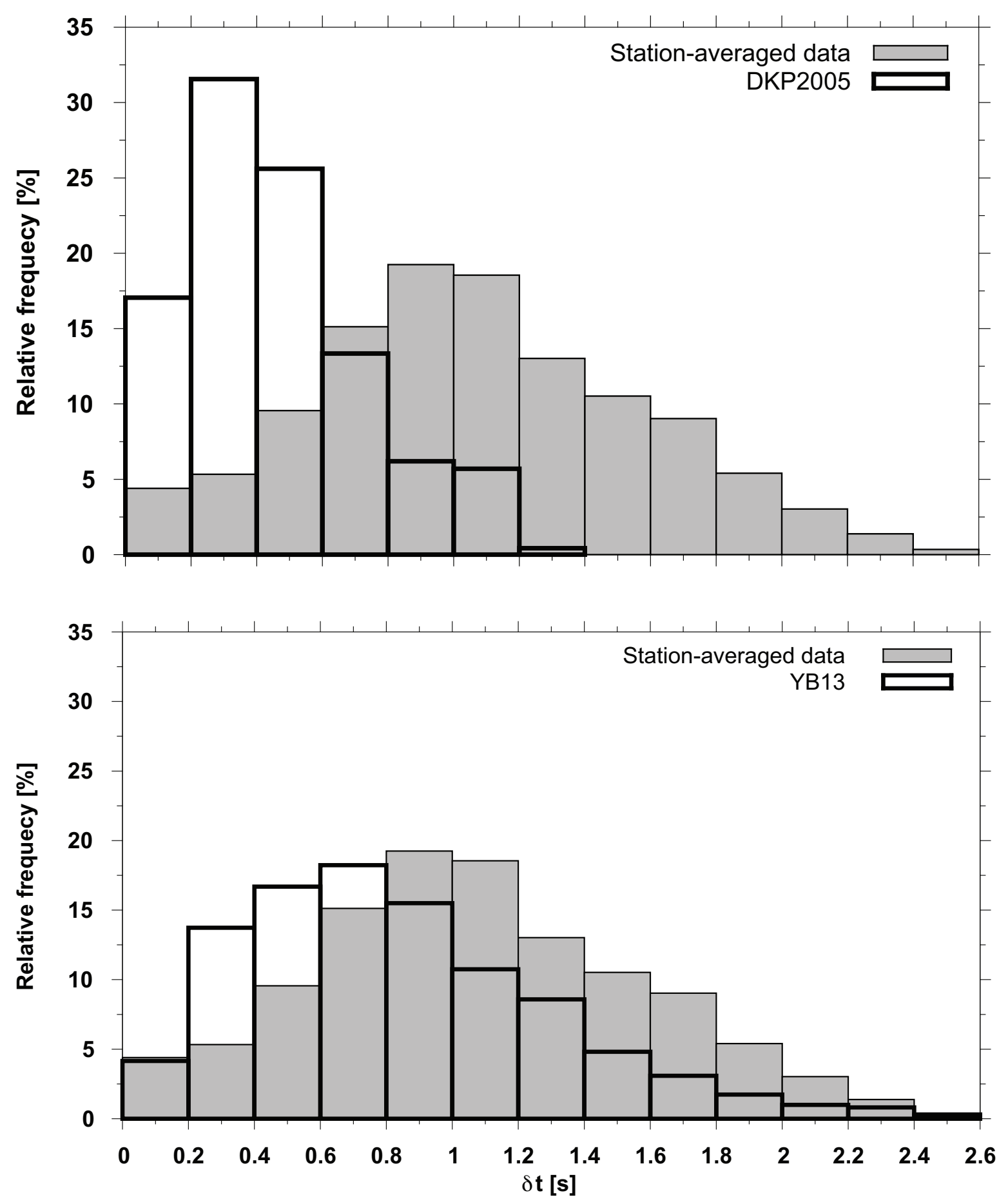

Figure 8: 

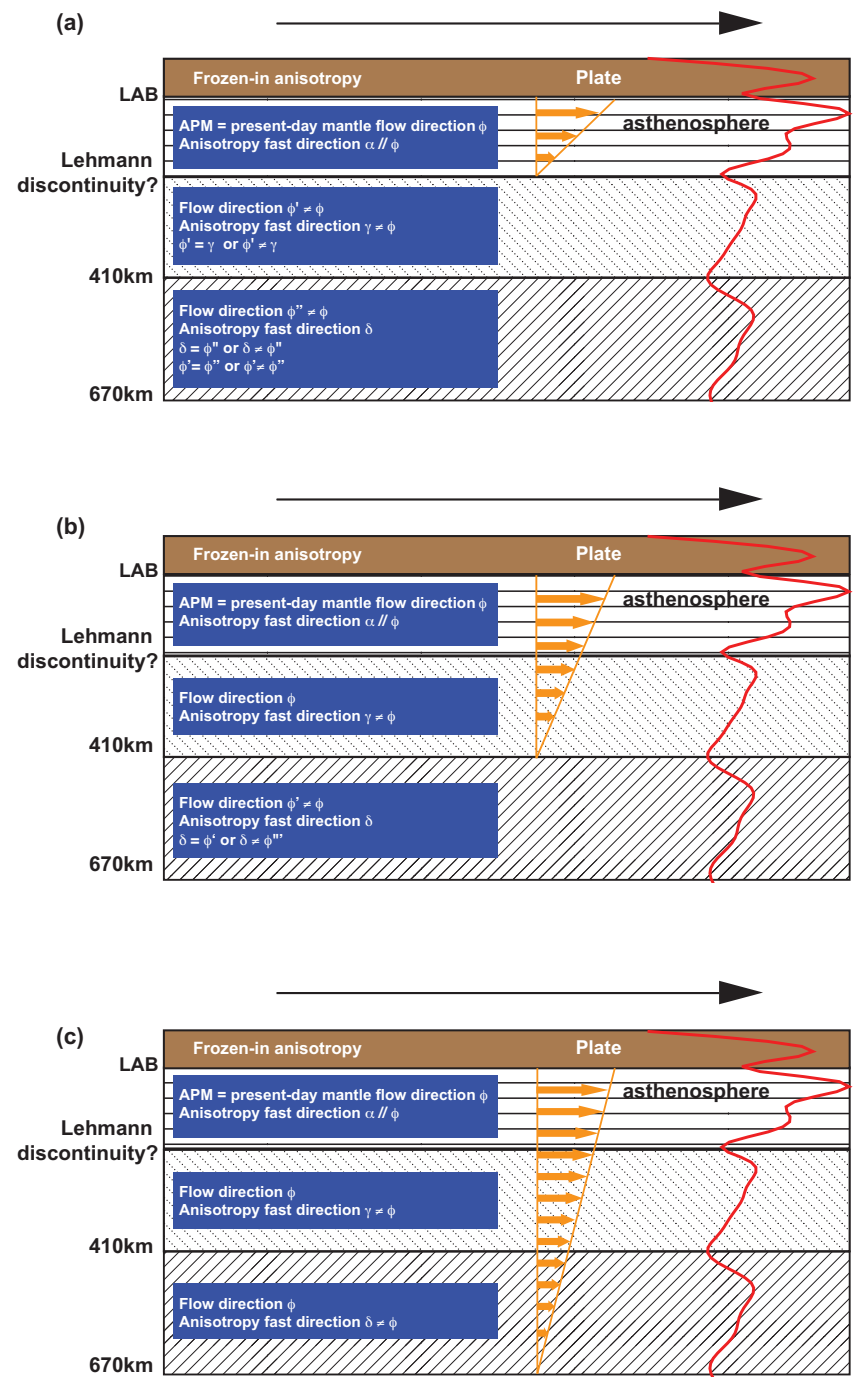

Figure 9: 


\begin{tabular}{|c|c|c|c|c|c|c|c|c|}
\hline & $\mathrm{n}=0$ & $\mathrm{n}=1$ & $\mathrm{n}=2$ & $\mathrm{n}=3$ & $\mathrm{n}=4$ & $\mathrm{n}=5$ & $\mathrm{n}=6$ & $\mathrm{n}=0-6$ \\
\hline YB13 & 0.858 & 0.895 & 0.861 & 0.827 & 0.802 & 0.698 & 0.680 & 0.856 \\
\hline model 1 & 0.800 & 0.748 & 0.736 & 0.709 & 0.643 & 0.615 & 0.417 & 0.742 \\
model 2 & 0.840 & 0.835 & 0.808 & 0.800 & 0.756 & 0.663 & 0.501 & 0.804 \\
\hline
\end{tabular}

Table 1: 